\title{
Genetic control of the number of grains on corn cob
}

\author{
S.A. Zaitsev*, D.P. Volkov, S.A. Guseva, and D.D. Babushkin
}

FSBSI Russian Research and Design-Technological Institute of Sorghum and Corn "Rossorgo", 1-y Institutsky proezd Str., 4, 410050 Saratov, Russia

\begin{abstract}
The evaluation of the combinational ability of maize I-lines was carried out on the basis of "the number of grains on the cob". This parameter is one of the significant components of the structure of plant productivity and harvest and is directly dependent on the number of grain rows and the number of grains in a cob row. The quantitative manifestation of this trait is significantly influenced by environmental conditions. A negative correlation of the studied trait with the seed size was revealed. Growing conditions strongly affect the value of the studied trait. The source material for breeding works with low and high indicators of combinational ability in terms of the number of grains on the cob is determined. Overdomination has a predominant effect in the manifestation of the number of grains on the cob. The values of the H1 dominance component are significant and notably higher than the values of the D component, which characterizes the variability caused by the additive action of genes. The values of the $\mathrm{H} 2 / 4 \mathrm{H} 1$ component indicate an uneven distribution among the parental lines of alleles with positive and negative effects. The number of genes or gene groups that control the number of grains on the cob varies from 2 to 3 . In 2016-2018, the paratypical variance component $(E)$ had a significant influence on the formation of the number of grains.
\end{abstract}

\section{Introduction}

A significant share in the breeding process is occupied by the breeding and genetic study of quantitative traits. Many of them have a polygenic basis and the patterns of their manifestation are of some interest [1]. In order to obtain the most accurate information about the combinational ability (CA), diallelic crosses are used [2]. It should be noted that CA manifestation is influenced by plant growing conditions. At the same time, the dependence of the common combinational ability on fluctuations in soil and climatic conditions of the environment is less than the specific one. To identify accurate SCA indicators, the study should be carried out in several ecological and geographical regions and over several years. The yield of corn grain products per unit area is determined by various components, one of them is the number of grains on the cob. This parameter

\footnotetext{
* Corresponding author: zea_mays@mail.ru
} 
directly depends on the appearance of the traits "number of grain rows" and "number of grains in a cob row" $[3,4,5]$. The quantitative manifestation of this trait is significantly influenced by environmental conditions: availability of moisture and nutrients, which affect plant ontogenesis during flowering. The lack of moisture supply due to arid conditions, the infestation of crops with weeds that limit the feeding area, contribute to the lag in cob development from panicle. The consequence of the resulting time interval between the flowering of panicle and cob is the deterioration of plant pollination and the formation of an unseeded rye ear.

Pollination conditions (fertilization) also deteriorate due to the pollen sterilitycaused by high temperature and low relative humidity

In some experiments, the role of additive effects in comparison with non-additive ones in the control of the studied trait was revealed. [6]. Research of the CA lines study for the number of grains on the cob in order to select for yield brought a tangible result to the development of the selection process methodology.

\section{The purpose of the study}

was to determine the degree of differences in the CCA and SCA effects in genetically homozygous breeding material (homozygous corn lines) by the parameter "number of grains on the cob".

\section{Material and methods}

The geographical area of the study (the Right Bank of the Saratov region) is characterized by a sharply continental climate. The hydrothermal coefficient (HTC) varies between: 1.201.45 (wet years); 0.70-0.95 (medium-wet years); 0.60-0.68 (dry years). The amount of falling moisture on average per year is $-360-455 \mathrm{~mm}$. The soil of the experimental site is southern low-humus medium-thick heavy-loamy chernozem. The soil density is $1.20-1.32$ $\mathrm{g} / \mathrm{cm} 3$, the lowest water capacity (LWC) of the $0-30 \mathrm{~cm}$ layer is $101.1 \mathrm{~mm}$, the $0-100 \mathrm{~cm}$ layer is $295.6 \mathrm{~mm}$, the permanent wilting point of plants (PWP) is $36.3 ; 151.4 \mathrm{~mm}$, respectively.

The experiment involved 11 self-pollinated corn lines, as well as their hybrid combinations obtained in the system of diallel crosses (method 2, model 1 according to Griffing). The study was carried out in 3-fold repetition with an accounting plot area of 7.7 $\mathrm{m}^{2}$. The standing density was formed manually in the phase of 4-5 leaves and amounted to 45 thousand plants/ha. To identify the CCA and SCA variances effects, appropriate statistical methods of genetic analysis were used [7, 8, 9]. The crop management included plowing, harrowing, cultivation, sowing with the SKS-6-10 cassette planter, and 2 row-torow treatments.

\section{Research results}

Such a complex parameter as "number of grains on the cob" consists of "number of grain rows" and "number of grains in a row" and is a component element of plant productivity and gross output. In addition, the expression of the studied indicator is influenced by the soil and climatic situation during the study. For example, in corn lines, the value of the trait varied within the following limits: in 269.0-541.3 pcs. (2016), 181.7-322.5 pcs. (2017), 260.4-464.1 pcs. (2018), which indicates the average variation degree (Table 1). A low variation coefficient in the number of grains was recorded on average for hybrids $(\mathrm{V}=5.1$ 9.2\%). This is confirmed by the data on the average group indicators of hybrids: 436.9- 
508.7 pcs. (2016), 307.0-404.4 pcs. (2017), 477.8-591.3 pcs. (2018). Ranking by the number of grains on the cob allowed to identify a certain stability in the years of study in the lines - IKV 18, Kd 12 L 53, V 29, RSK3, RSK 218, and also to arrange them depending on the average yield in the following sequence: RSK $3<$ RSK $7<$ V $47<$ IKV $18<$ V $27<$ V $29<$ V $117<$ RSK $218<$ V $72<$ IS $134<$ Kd 12 L 53.

Judging by the average data (Table 1), when crossing self-pollinated corn lines, there is a significant heterosis in the number of grains on the cob, especially in favorable conditions for the sporophytes formation. Thus, in 2016, heterosis on the studied trait was $24.3 \%$, in $2017-34.4 \%$, in $2018-45.7 \%$.

Table 1. Indicators of the grain number on the cob of I-lines $\left(\mathrm{P}^{*}\right)$ and the average group values of hybrids $\left(\mathrm{F} 1^{* *}\right)$, pcs.

\begin{tabular}{|c|c|c|c|c|c|c|}
\hline \multirow{2}{*}{ Line } & \multicolumn{2}{|c|}{2016} & \multicolumn{2}{|c|}{2017} & \multicolumn{2}{|c|}{2018} \\
\hline & $\mathrm{P} *$ & $\mathrm{~F}_{1} * *$ & $\mathrm{P}^{*}$ & $\mathrm{~F}_{1} * *$ & $\mathrm{P}^{*}$ & $\mathrm{~F}_{1} * *$ \\
\hline V 47 & 381.0 & 501.7 & 192.8 & 367.4 & 317.0 & 510.2 \\
\hline IKV 18 & 337.8 & 508.1 & 237.6 & 404.4 & 321.5 & 591.3 \\
\hline V 27 & 417.7 & 436.9 & 244.7 & 327.0 & 292.6 & 517.2 \\
\hline V72 & 365.0 & 508.2 & 315.5 & 307.0 & 402.7 & 527.3 \\
\hline Kd 12 L 53 & 541.3 & 500.3 & 322.5 & 322.4 & 394.5 & 503.0 \\
\hline V29 & 387.0 & 455.2 & 250.9 & 315.6 & 358.9 & 477.8 \\
\hline V 117 & 451.7 & 483.0 & 281.3 & 336.3 & 341.1 & 496.8 \\
\hline IS 134 & 392.4 & 508.7 & 239.1 & 360.6 & 464.1 & 505.4 \\
\hline RSK 3 & 269.0 & 457.3 & 181.7 & 313.7 & 298.4 & 482.6 \\
\hline RSK 218 & 392.1 & 479.0 & 277.2 & 334.4 & 405.3 & 507.0 \\
\hline RSK 7 & 345.2 & 482.1 & 261.9 & 381.4 & 260.4 & 498.8 \\
\hline$\underline{\bar{X}}$ & 389.1 & 483.7 & 255.0 & 342.7 & 350.6 & 510.7 \\
\hline$F_{\text {fact }}$ & $3.5^{*}$ & $6.8^{*}$ & $4.3^{*}$ & $5.7 *$ & $5.1 *$ & $7.2^{*}$ \\
\hline $\mathrm{LSD}_{0.05}$ & 102.1 & 18.0 & 68.1 & 38.7 & 74.3 & 20.3 \\
\hline $\mathrm{V}, \%$ & 17.7 & 5.1 & 17.3 & 9.2 & 17.3 & 5.9 \\
\hline
\end{tabular}

The nature of the correlation between the grain yield and the grain number on the cob can be positive and significant or insignificant [10]. The inconsistency of the data can be explained by the influence of abiotic factors or genetic modifications on the phenotypic manifestation of crop structure elements. These changes are possible to the point where they become factors that determine or limit the yield. The nature of the relationship between the grain yield and the grain number on the cob is also influenced by complex interactions between the components of the yield structure. Thus, a positive correlation was registered between the elements "grain number on a cob" and "number of grain rows", "number of grains in a row", while the parameter "weight of 1000 grains" correlates negatively with the studied trait (Table 2). The correlation coefficients between the grain number on the cob and the other quantitative characteristics studied were insignificant.

Table 2. Correlation coefficients between the "grain number on the cob" trait and other quantitative plant traits in lines (P) and hybrids (F1)

\begin{tabular}{|l|c|c|c|c|c|c|}
\hline \multirow{2}{*}{ Correlating trait } & \multicolumn{2}{|c|}{2016} & \multicolumn{2}{|c|}{2017} & \multicolumn{2}{c|}{2018} \\
\cline { 2 - 7 } & $\mathrm{P}^{*}$ & $\mathrm{~F}_{1}^{* *}$ & $\mathrm{P}^{*}$ & $\mathrm{~F}_{1}{ }^{*}$ & $\mathrm{P}^{*}$ & $\mathrm{~F}_{1} *^{*}$ \\
\hline Stem length & 0.52 & -0.20 & 0.38 & 0.33 & 0.48 & 0.43 \\
\hline Cob initiation height & 0.38 & 0.41 & $0.68^{*}$ & 0.19 & 0.48 & $0.66^{*}$ \\
\hline Cob length & $0.61^{*}$ & 0.28 & $0.60^{*}$ & 0.42 & 0.43 & 0.20 \\
\hline Length of grained part of the cob & 0.56 & 0.51 & 0.48 & $0.83^{* *}$ & 0.36 & 0.42 \\
\hline Cob thickness & 0.28 & -0.03 & 0.32 & -0.35 & 0.49 & 0.11 \\
\hline Number of grain rows & 0.53 & 0.57 & $0.73^{*}$ & 0.18 & $0.72^{*}$ & $0.79^{* *}$ \\
\hline Number of grains in a row & 0.64 & $0.67^{*}$ & $0.94^{* *}$ & $0.81^{* *}$ & $0.91^{* *}$ & $0.85^{* *}$ \\
\hline Weight of 1000 grains & $-0.79^{* *}$ & -0.53 & -0.04 & $-0.64^{*}$ & -0.30 & $-0.78^{* *}$ \\
\hline Grain yield & 0.62 & 0.32 & $0.81^{* *}$ & $0.75^{* *}$ & 0.55 & 0.05 \\
\hline
\end{tabular}


To predict the trait manifestation in a certain range of external conditions, it is necessary to evaluate the CCA effects. The calculation of the data on the studied trait allowed to identify the effects of the common combining ability and variance of the specific combining ability (Table 3.). The high CCA effect was distinguished by the sample of IKV 18 in 2016, 2018, and also the greatest SCA dispersion was noted in IKV 18. RSK 3 was characterized by a low CCA effect in 2016-2018. The remaining lines differed in the average value of the CCA effect within different limits.

During the study, a high SCA effect was found in the parameter "grain number on the cob" in the system of diallelic crosses of hybrid combinations in the combinations of crosses: in 2016 - Kd 12 L 53 / V 47, RSK 3 / IKV 18, RSK 218 / V 47, V 27 / IKV 18, V 117 / V 27, IS 134 / V 72, IS 134 / V 29, RSK 7 / V 117, RSK 7 / IS 134; in 2007 - Kd 12 L53 / V B47, RSK 218 / V 47, V 27 / IKV 18, V 117 / IKV 18, RSK 218 / IKV 18, Kd 12 L 53 / V 72, RSK 3 / IS 134, RSK 7 / RSK 3; in 2018 - IS 134 / V 47, RSK 218 / V 47, Kd 12 L 53 / IKV 18, RSK / IKV 18, RSK 218 / IKV 18, RSK7 / IKV 18, RSK 218 / V 27, V 117 / V 72.

Table 3. CCA effect and SCA line variance

\begin{tabular}{|c|c|c|c|c|c|c|}
\hline \multirow{2}{*}{ Line } & \multicolumn{2}{|c|}{2016} & \multicolumn{2}{c|}{2017} & \multicolumn{2}{c|}{2018} \\
\cline { 2 - 7 } & $\begin{array}{c}\text { CCA } \\
\text { effect }\end{array}$ & $\begin{array}{c}\text { SCA } \\
\text { variance }\end{array}$ & $\begin{array}{c}\text { CCA } \\
\text { effect }\end{array}$ & $\begin{array}{c}\text { SCA } \\
\text { variance }\end{array}$ & $\begin{array}{c}\text { CCA } \\
\text { effect }\end{array}$ & $\begin{array}{c}\text { SCA } \\
\text { variance }\end{array}$ \\
\hline V 47 & 3.1 & 2395.2 & 26.2 & 3103.7 & -5.5 & 4337.4 \\
\hline IKV 18 & 43.5 & 4020.7 & 10.7 & 4045.5 & 57.5 & 9255.8 \\
\hline V 27 & -15.0 & 3034.8 & -31.8 & 1358.2 & -3.9 & 4310.7 \\
\hline V 72 & -3.0 & 2423.6 & 25.2 & 1031.2 & 20.8 & 4745.7 \\
\hline Kd 12 L 53 & -6.6 & 2882.6 & 25.2 & 3732.0 & 0.9 & 5165.0 \\
\hline V 29 & -22.7 & 2025.6 & -22.5 & 1704.0 & -24.0 & 2914.5 \\
\hline V 117 & -2.2 & 2301.0 & 8.9 & 2972.0 & -12.1 & 6870.0 \\
\hline IS 134 & 10.0 & 3532.8 & 19.5 & 3066.9 & 13.4 & 5394.8 \\
\hline RSK 3 & -35.0 & 3607.6 & -49.8 & 5203.5 & -29.6 & 3264.9 \\
\hline RSK 218 & -1.7 & 3585.4 & -3.4 & 1381.5 & 5.6 & 7802.1 \\
\hline RSK 7 $_{\text {RS }}$ & 29.5 & 2663.3 & -8.2 & 2947.9 & -23.0 & 5498.0 \\
\hline F $_{\text {fact }}$ & $6.7 *$ & $3.5^{*}$ & $8.1 *$ & $6.0 *$ & $14.7 *$ & $17.9^{*}$ \\
\hline LSD $_{0.05}$ & 30.7 & & 39.3 & & 25.5 & \\
\hline
\end{tabular}

When calculating the Hayman data, it was found that the manifestation of epistasis in some lines does not allow to correctly apply this method. The calculation of the genetic variance components showed that the additive-dominant model becomes adequate when the RSK 3 line is removed from the calculations in 2016 and the lines V-47, V-18, V-27, and V-72 in 2018 (Table 4). This proves the absence of non-allelic interactions in the inheritance scheme for the remaining lines of the diallelic scheme and increases the objectivity of the Heyman analysis. A rather strong variation of the Fr parameter was revealed. This component indicates the relative contribution of dominant and recessive genes to the development of the trait in hybrids of each self-pollinated line, and also characterizes the dominance direction. A significant negative value of the Fr parameter is observed at the RSK 7 line in 2018. This side of the results indicates the absence of dominant genes of positive action in the line. According to the lines Kd 12 L 53, V 29, IS 134, RSK 218 a manifestation of dominance was revealed, which is directed towards increasing the number of grains on the cob. 
Table 4. Values of the Fr parameter of the lines for the trait "grain number on the cob"

\begin{tabular}{|l|c|c|c|}
\hline \multicolumn{1}{|c|}{ Component } & 2016 & 2017 & 2018 \\
\hline Fr V 47 & $-6117.3 \pm 3731.5$ & $-232.3 \pm 3357.6$ & epistasis \\
\hline Fr IKV 18 & $-3053.5 \pm 3731.5$ & $-1601.1 \pm 3357.6$ & epistasis \\
\hline Fr V 27 & $7762.7 \pm 3731.5$ & $28.1 \pm 3357.6$ & epistasis \\
\hline Fr V 72 & $6265.7 \pm 3731.5$ & $1142.8 \pm 3357.6$ & epistasis \\
\hline Fr Kd 12 L 53 & $-599.0 \pm 3731.5$ & $2890.5 \pm 3357.6$ & $16513.1 \pm 6349.8^{*}$ \\
\hline Fr V 29 & $8430.0 \pm 3731.5^{*}$ & $5780.9 \pm 3357.6$ & $10635.4 \pm 6349.8$ \\
\hline Fr V 117 & $5654.2 \pm 3731.5$ & $2475.9 \pm 3357.6$ & $2225.2 \pm 6349.8$ \\
\hline Fr IS 134 & $6557.5 \pm 3731.5$ & $6393.2 \pm 3357.6$ & $18821.5 \pm 6349.8^{*}$ \\
\hline Fr RSK 3 & epistasis & $-121.1 \pm 3357.6$ & $2946.5 \pm 6349.8$ \\
\hline Fr RSK 218 & $9387.9 \pm 3731.5^{*}$ & $1426.3 \pm 3357.6$ & $18235.0 \pm 6349.8^{*}$ \\
\hline Fr RSK 7 & $-2269.4 \pm 3731.5$ & $-5297.9 \pm 3357.6$ & $-13515.7 \pm 6349.8^{*}$ \\
\hline
\end{tabular}

It should be noted that there is a negative correlation between the indicator of the parameter "grain number on the cob" and the dominance of the parental lines, which was $r$ $=-0.17(\mathrm{df}=8)$ in 2016; $\mathrm{r}=-0.27(\mathrm{df}=9)$ in 2017; and $\mathrm{r}=-0.92(\mathrm{df}=5)$ in 2018. The presence of negative correlation values $(r)$ suggests that there is a relationship between the number of grains on the cob and the presence of dominant alleles in the parental forms. Thus, lines with a large number of grains on the cob have the largest number of dominant alleles. Apparently, the dominant alleles determine the increase in the trait, and the recessive ones determine its decrease, i.e. in this case, the number of grains on the cob is controlled by the dominant alleles.

According to the biometric-genetic method $\left(\sqrt{\mathrm{H}_{1}} / \mathrm{D}>1\right)$, the conclusion is that the manifestation of the grain number on the cob mainly depends on the influence of the overdomination effect (Table 5). During the study, the value of the $\mathrm{H}_{1}$ dominance component was significant and notably higher than that of the $\mathrm{D}$ component, which characterizes the variability caused by the additive action of genes. On average, for all hybrids, the dominance is directed towards increasing the number of grains on the cob. The values of the $\mathrm{F}$ component, which reflects the contribution of dominant and recessive effects on the manifestation of the trait, are insignificant in all cases, which indicates an approximately equal ratio between dominant and recessive genes. This is confirmed by the values of the relations $\sqrt{ }\left(4 \mathrm{DH}_{1}\right)+\mathrm{F} / \sqrt{ }\left(4 \mathrm{DH}_{1}\right)$-F equal to one.

The values of the $\mathrm{H}_{2} / 4 \mathrm{H}_{1}$ component are less than the theoretical one $(0.25)$, which means that the plus and minus alleles are not distributed symmetrically between the lines. The number of genes or gene groups that control the number of grains on the cob varies from 2 to 3. In 2016-2018, the paratypical variance component (E) had a significant influence on the formation of the number of grains.

Table 5. Components of the genetic variance by the number of grains on the cob

\begin{tabular}{|c|c|c|c|}
\hline Component & 2016 & 2017 & 2018 \\
\hline $\mathrm{D}$ & $2216.7 \pm 1083.1^{*}$ & $842.3 \pm 973.7$ & $4628.7 \pm 1852.0^{*}$ \\
\hline $\mathrm{F}$ & $3201.9 \pm 2499.1$ & $2134.7 \pm 2225.4$ & $7980.2 \pm 4442.9$ \\
\hline $\mathrm{H}_{1}$ & $11083.6 \pm 2305.5^{*}$ & $14815.6 \pm 2006.3$ & $20928.9 \pm 4458.7^{*}$ \\
\hline $\mathrm{H}_{2}$ & $7986.8 \pm 1959.4^{*}$ & $11072.7 \pm 1686.6^{*}$ & $16460.4 \pm 3928.7^{*}$ \\
\hline $\mathrm{h}$ & $21601.5 \pm 1311.6^{*}$ & $24563.5 \pm 1128.2^{*}$ & $29645.62 \pm 2638.7^{*}$ \\
\hline $\mathrm{E}$ & $2942.3 \pm 326.6^{*}$ & $2159.2 \pm 281.1^{*}$ & $1781.1 \pm 654.8^{*}$ \\
\hline $\mathrm{m} 11-\mathrm{m} 10$ & 75.44 & 79.60 & 87.52 \\
\hline$\sqrt{\mathrm{H}_{1} / \mathrm{D}}$ & 2.24 & 8.79 & 2.13 \\
\hline $\mathrm{H}_{2} / 4 \mathrm{H}_{1}$ & 0.18 & 0.19 & 0.20 \\
\hline$\left.\sqrt{(4 \mathrm{DH}} \mathrm{H}_{1}\right)+\mathrm{F} /$ & 1.00 & 1.00 & 1.00 \\
\hline$\sqrt{(4 \mathrm{DH}})-\mathrm{F}$ & & 2.22 & 1.80 \\
\hline $\mathrm{h} / \mathrm{H}_{2}$ & 2.70 & & \\
\hline
\end{tabular}




\section{Conclusions}

The use of diallel analysis makes it possible to determine the components of the genetic variance and to evaluate the self-pollinated corn lines included in the working collection. Even taking into account the incomplete compliance with all the requirements of the B.I. Hayman method, the information obtained on the inheritance scheme of a breedingvaluable trait and on the variability of inheritance in ecological conditions is of scientific and practical interest. Growing conditions have a modifying effect on the system of genetic control of the number of grains on the cob, both in a certain group of lines and in individual parental forms. Nevertheless, a pattern is clearly evident - overdomination plays a predominant role in the control of the studied trait. Some significant contribution to the control of the grains number on the cob is also made by genes that exhibit an additive effect. The effect of epistasis on the trait manifestation is insignificant. An increase in the grains number on the cob in hybrids is not always associated with the positive effect of dominant genes. In the breeding process, it is advisable to select the studied trait in later generations of inbreeding, with the accumulation of homozygotes. There is a real possibility of breeding of with an increased number of grains on the cob, since genes with an additive effect take part in the control of the studied trait and transgression is possible.

\section{References}

1. P.P. Litun, Selection and seed production, 56, 40 (1984)

2. V.I. Zhuzhukin, Success of modern Natural Science, 10, 50 (2018)

3. N.A. Orlyanskiy, Corn and sorghum, 3, 3 (2019)

4. A.L. Zozulya, Work on applied Botany, Genetics and Breeding, 69(1), 116 (1980)

5. E.M. Govor, Proceedings on Applied Botany, Genetics and Breeding, 181(2), 28 (2020)

6. G.I. Vedeneev, abstract of the dissertation of the Doctor of agricultural Sciences: 06.01.05, 48 (1990)

7. M.A. Fedin, Statistical methods of genetic analysis, 208 (1980)

8. B. Griffing, J. Biol. Sci., 9, 463 (1956)

9. B.I. Hayman, Genetics, 10, 235 (1954)

10. V.S. Ilyin, Success of modern Natural Studies, 11(1), 43 (2016) 